\title{
Interstellar oxygen chemistry
}

\author{
S. Viti ${ }^{1}$, E. Roueff ${ }^{2}$, T. W. Hartquist ${ }^{3}$, G. Pineau des Forêts ${ }^{2}$, and D. A. Williams ${ }^{1}$ \\ 1 Department of Physics and Astronomy, UCL, Gower St., London WC1E 6BT, UK \\ 2 DAEC and UMR8631, Observatoire de Paris, Place J. Janssen, 92190 Meudon, France \\ 3 Department of Physics and Astronomy, University of Leeds, Leeds LS2 9JT, UK
}

Received 3 November 2000 / Accepted 9 February 2001

\begin{abstract}
We present results of chemical models for a variety of types of regions shown by SWAS observations to contain less $\mathrm{O}_{2}$ and $\mathrm{H}_{2} \mathrm{O}$ than previously expected. We identify time-dependent models for which $\mathrm{O}_{2}$ and $\mathrm{H}_{2} \mathrm{O}$ abundances meet the SWAS constraints and for which calculated abundances of other species are in harmony with measurements made primarily at millimeter wavelengths. The phases of acceptable composition are transient in these time-dependent models but are of very substantial length in many models for which $\mathrm{CO}$ and $\mathrm{N}_{2}$ are assumed to be returned promptly and unaltered to the gas phase though other species, except for $\mathrm{H}_{2}$ and He, freeze-out onto the dust. We also consider whether the presence of bistability in some steady-state models can account for the SWAS and other observations.
\end{abstract}

Key words. ISM: abundances - ISM: clouds - ISM: molecules

\section{Introduction}

It has often been asserted that water and molecular oxygen are important reservoirs of the elemental oxygen in the ISM. It is also frequently assumed that they provide major sources of cooling. Atmospheric absorption impedes ground based observations of both of these species and it was not until the advent of SWAS (Submillimeter Wave Astronomy Satellite) that direct detection of likely components of the gas phase oxygen reservoir in dense cores could be made. SWAS has been used in pointed observations of the ground state ortho- $\mathrm{H}_{2} \mathrm{O}$ and low energy $\mathrm{O}_{2}$ transitions towards regions of high and low mass star formation and several clouds with little or no star formation. These observations have given severe constraints for theoretical models of interstellar clouds (see the special issue of ApJL, Vol. 539, Part II). On the other hand, ISO - SWS observations (Vandenbussche et al. 1999) have provided other constraints on the abundance of solid $\mathrm{O}_{2}$ which accounts less than $6 \%$ of the total oxygen budget in the dense interstellar clouds.

SWAS data show that in the Orion Molecular Cloud, gaseous water and $\mathrm{O}_{2}$ are not the main reservoirs of elemental oxygen. Their abundances account for much less than $1 \%$ of the total abundance of oxygen; the total is constrained by observations of diffuse clouds where the fractional abundance of all the oxygen not locked in grains (in silicates) is of the order of $\sim 3.210^{-4}$. In dense regions of

Send offprint requests to: S. Viti, e-mail: sv@star.ucl.ac.uk interstellar space, however, a large portion of the oxygen is found on grains as water ice.

Contrary to model predictions (e.g. Maréchal et al. 1997), the SWAS results also indicate that $\mathrm{O}_{2}$ and $\mathrm{H}_{2} \mathrm{O}$ are not the major coolants in general for molecular clouds, although in some localized regions, such as shocked, hot regions, ISO has shown that the gas-phase fractional abundance of water can be as high as $\sim 10^{-4}$ (Harwit et al. 1998; Nisini et al. 2000).

Bergin et al. (2000) summarized the constraints yielded by SWAS and ISO as follows:

(1) regardless of the line of sight, the fractional abundance relative to hydrogen nuclei of gaseous molecular oxygen, $X\left(\mathrm{O}_{2}\right)$, in dense molecular clouds is less than $\sim 10^{-6}$;

(2) in starless cores (such as core B in TMC-1) the fractional abundance of $\mathrm{H}_{2} \mathrm{O}, X\left(\mathrm{H}_{2} \mathrm{O}\right)$, is less than $710^{-8}$;

(3) in low density regions, such as those along the line of sight towards Sgr B2, gaseous water is relatively abundant $\left(X\left(\mathrm{H}_{2} \mathrm{O}\right) \sim 10^{-6}\right)$ while $\mathrm{O}_{2}$ is not detected; $X\left(\mathrm{O}_{2}\right)<10^{-6}$. The fractional abundance of water found by SWAS along the line of sight towards Sgr B2 also confirms previous ISO results (Moneti et al. 2001). If low temperature gas phase chemistry is responsible for the gaseous $\mathrm{H}_{2} \mathrm{O}$, this result is puzzling as molecular oxygen and water formation are coupled in the standard gas-phase chemistry: once $\mathrm{H}_{3}^{+}$reacts with neutral oxygen, rapid reactions will lead to the formation of the ion $\mathrm{H}_{3} \mathrm{O}^{+}$. The latter dissociatively 
recombines with electrons to form, among other species, $\mathrm{OH}$ and $\mathrm{H}_{2} \mathrm{O}$. $\mathrm{OH}$ then reacts with atomic oxygen to form molecular oxygen; if $\mathrm{H}_{2} \mathrm{O}$ is abundant, one would expect $\mathrm{O}_{2}$ to be abundant also;

(4) in high density star formation regions gaseous $X\left(\mathrm{H}_{2} \mathrm{O}\right)$ is $\sim 10^{-8}$;

(5) the fractional abundance of water ice is measured to be $\sim 10^{-4}$ in dense cores $\left(10^{3} \leq n_{\mathrm{H}} \mathrm{cm}^{-3} \leq 10^{6}\right.$ where $n_{\mathrm{H}}$ is the number density of hydrogen nuclei; $T_{\text {gas }}<$ $50 \mathrm{~K}$ ) (Shutte 1999).

Bergin et al. remarked that the low abundance of water in star formation regions cannot be accounted for by rapid photodissociation by intense UV radiation fields as other species, such as $\mathrm{NH}_{3}$, would also be destroyed. Such species are however observed to be abundant.

The constraints imposed by SWAS (and ISO) represent a challenge for theorists. Why do simple models predict large abundances at steady-state for gaseous $\mathrm{H}_{2} \mathrm{O}$ and $\mathrm{O}_{2}$ ? Where is the oxygen in quiescent clouds, and in regions of low-mass and high-mass star formation? Chemical models must self-consistently reproduce: (i) the gas-phase molecular oxygen and water abundances observed with SWAS; (ii) the water ice abundance measured with ISO; (iii) the abundances of simple and complex oxygen and carbon bearing species observed with ground based telescopes, and (iv) the observed abundances in a variety of different environments such as regions of high and low mass star formation and starless cores. We note, however, that the SWAS beam is as large as 4 arcmin and that the SWAS observations inevitably encompass a variety of interstellar and circumstellar regions; in effect, the SWAS results give averages over a variety of conditions.

In this paper we explore two theoretical approaches which may provide solutions to the constraints imposed by SWAS while still satisfying the constraints of observations of other simple and complex species. Section 2 is dedicated to the first approach: we investigate time-dependent models where gas phase species are allowed to deplete on to the grains. Bergin et al. (2000) showed that the SWAS data can be explained with low temperature gas phase models only if the observed clumps are short-lived, and we explore this point in detail. We show that "transient" states in which the chemistry satisfies constraints arising from SWAS and other observations are very long-lived in models where most species freeze-out efficiently on to dust grains except for $\mathrm{CO}$ and $\mathrm{N}_{2}$ which are returned promptly to the gas phase. Section 3 concerns the applicability of bistability solutions to the interpretation of the SWAS results. The gas phase chemistries discussed in Sects. 2 and 3 are identical. Section 4 presents a discussion of our findings.

\section{Time-dependent chemical models}

\subsection{The model assumptions}

In each of the models that we consider in this section physical and chemical conditions match those inferred from observations during one epoch only; at other times, the physical and chemical conditions in each model are such that the source is undetectable with the sorts of observations already made. Cyclic models of the kind originally suggested by Norman \& Silk (1980) have the potential to provide such transient observable conditions (e.g. Williams \& Hartquist 1984; Charnley et al. 1988). In this category of models, it is assumed that interstellar and circumstellar gas is continually re-cycled from low to high density, probably by the action of star formation itself. This approach has some attractions, given that the link between interstellar chemistry and dynamics has been frequently discussed.

We model the chemistry of regions of high and low mass star formation and starless cores. For ease of computation a cloud is represented as a single point within a semi-infinite slab with a pseudo-time-dependent chemical simulation. The UMIST chemical database (Millar et al. 1997) was used with some modifications (see Viti et al. 2000), particularly with regard to the gas-grain interaction. In some models, we have included the freeze-out of all gas phase species, except hydrogen and helium, on to dust grains. All the species that deplete on to grains are incorporated in mantles and it is assumed that they are never released. The modified rate file contains 397 species taking part in 4246 reactions (including freeze-out reactions). In all models, except the oxygen poor ones (see later), the initial elemental abundances by number relative to hydrogen were taken to be $7.010^{-2}, 2.210^{-4}, 6.010^{-5}$, $4.610^{-4}, 1.310^{-5}, 7.010^{-9}, 2.010^{-9}$ and $8.010^{-9}$ for helium, carbon, nitrogen, oxygen, sulphur, magnesium, sodium, and silicon respectively. As an initial condition we also assumed that half the elemental abundance of carbon is locked in the carbonaceous grains. Our models are time-dependent; this implies that the depletion of each element was not assumed but rather, was derived through a computation of the non-steady state chemical evolution of the gas/dust interaction processes.

We have performed computations for a grid of models. A model is characterized by whether (i) the gas is static or collapsing; the collapse is treated as a "modified" free-fall as defined by Rawlings et al. (1992) where the density was assumed to increase in a fashion governed by

$\frac{\mathrm{d} n_{\mathrm{H}}}{\mathrm{d} t}=B\left(\frac{n_{\mathrm{H}}^{4}}{n_{\mathrm{H} 0}}\right)^{1 / 3}\left\{24 \pi G m_{\mathrm{H}} n_{\mathrm{H}}\left[\left(\frac{n_{\mathrm{H}}}{n_{\mathrm{H} 0}}\right)^{1 / 3}-1\right]\right\}^{1 / 2}$

where $t, B, G$, and $m_{\mathrm{H}}$ are the time, a numerical constant, the gravitational constant, and the mass of a hydrogen atom respectively; in practice, we took $B=1$, which corresponds to a free-fall collapse. (ii) the final number density, $n_{\mathrm{H}}=n(\mathrm{H})+2 n\left(\mathrm{H}_{2}\right)$, is low $\left(10^{3} \mathrm{~cm}^{-3}\right)$, medium $\left(10^{4} \mathrm{~cm}^{-3}\right)$ or high $\left(10^{5}, 10^{6} \mathrm{~cm}^{-3}\right)$; (iii) the initial oxygen abundance is standard or low; (iv) the freeze-out efficiency of gas phase elements onto grains is zero, medium, or high; (v) the temperature is low $(10 \mathrm{~K})$ or medium $(30 \mathrm{~K})$; (vi) the visual extinction is moderate $(2 \mathrm{mag}$ ) or high (5 and $15 \mathrm{mag}$ ); (vii) the branching ratio for $\mathrm{H}_{3} \mathrm{O}^{+}$ 
dissociative recombination with electrons is higher or lower, according to recent determinations (see below).

Note that the initial density for the models where the final density reaches $10^{6} \mathrm{~cm}^{-3}$ is slightly higher $\left(10^{4}\right.$ instead of $10^{3} \mathrm{~cm}^{-3}$ ) than employed for the rest of the models where collapse occurs; this was necessary as, in a free-fall collapse from the lower initial density, the final densities are not reached until 1.7 Myr, by which time everything is already frozen on to the grains: this picture is unrealistic. We can justify our choice of having a slightly higher initial value of $n_{\mathrm{H}_{2}}$ if we think of a high mass star as the result of a denser small clump within a much bigger and less dense cloud.

The ambient interstellar radiation field was taken to be that defined by Habing (1968). For the models where collapse occurs, it was assumed that a clump collapses gravitationally within the molecular cloud and that the collapse is a free-fall. When we allowed freeze-out to occur during the collapse phase, gas-phase chemistry and freeze-out on to dust grains with subsequent processing, such as hydrogenation, were assumed to occur. For depletion, we used the parameter $F R$ where $F R=0$ implies no freeze-out and $F R=1$ means that sticking occurs on every collision. For most of the models we adopted the branching ratios for dissociative recombination of $\mathrm{H}_{3} \mathrm{O}^{+}$(main routes for oxygen chemistry) measured by Vejby-Christensen et al. (1997), but several selected models were also computed with the branching ratios measured by Williams et al. (1996). O'neill \& Williams (1999) and Bergin et al. (2000) noted that these two laboratory measurements produce very different amounts of water.

For our "oxygen poor" models we have halved the oxygen initial elemental abundance: the two initial abundances we chose for oxygen are justified by the interstellar oxygen abundances derived from 14 lines of sight by Meyer et al. (1998, see their Table 2). The oxygen abundance that we use as standard is taken from Snow \& Witt (1996), who presented elemental abundances derived from stars in the solar neighbourhood rather than from the Sun.

Our choice of initial physical conditions is based on the parameters suggested by Bergin et al. (2000) representing the various lines of sight observed with SWAS. A total of 67 models have been evaluated, so that the parameter space described here has been thoroughly explored. The results of some of these are presented in the discussion below. A further 30 models, in which higher cosmic ray ionization rates were adopted, were also evaluated but their results are not reported here as none are in harmony with the SWAS observations of the oxygen chemistry in dense cores.

\subsection{Models in which all massive species freeze-out}

Table 1 gives the parameters defining all the computational models we constructed. The fifth and sixth column indicate whether the cloud has undergone collapse (-c-) or is static (-nc-) and whether the initial abundances are standard (-s) or oxygen poor (-ns). In the seventh column, "No" indicates that $\mathrm{CO}$ and $\mathrm{N}_{2}$ are assumed to return instantaneously to the gas phase after freezing-out. The last column indicates whether we adopted the branching ratios measured by Vejby-Christensen et al. (1997, VB97) or the ones measured by Williams et al. (1996, W96). For our discussion we follow the constraints laid out in Bergin et al. (2000), Sect. 4. Generally, SWAS results impose an upper limit for the fractional abundance of molecular oxygen of $<10^{-6}$ (Goldsmith et al. 2000), averaged over the SWAS beam, in all regions surveyed. The models therefore need to be capable of satisfying that constraint, together with (i) $X\left(\mathrm{H}_{2} \mathrm{O}\right)<710^{-8}$ for starless cores, (ii) $X\left(\mathrm{H}_{2} \mathrm{O}\right) \sim 10^{-6}$ for low density gas, (iii) $10^{-9}<$ $X\left(\mathrm{H}_{2} \mathrm{O}\right)<$ few $\times 10^{-8}$ in high mass star forming regions.

In this subsection we consider models in which all species containing an element more massive than helium freeze out on collision with dust. We divide the remainder of this subsection into three parts.

\subsubsection{Starless cores or cores with little evidence of star-formation}

In cores with little evidence of star-formation such as TMC-1 core $\mathrm{B}\left(n_{\mathrm{H}} \geq 10^{4} \mathrm{~cm}^{-3} ; T_{\text {gas }}=10 \mathrm{~K} ; A_{V}>\right.$ $10 \mathrm{mag}) X\left(\mathrm{H}_{2} \mathrm{O}\right)$ is found to be $<710^{-8}$. For these environments, we excluded collapse (a plausible assumption for starless cores). In some models we included freeze out but never with total efficiency. In reality some depletion must occur as the fractional abundance of water ice is observed to be high $\left(\sim 10^{-4}\right)$ also in starless cores. From the grid, Models 1 to 4 were considered to represent starless cores, and Model 3 was found to be the "best matching" model (defined as the one that best reproduced the observed water and molecular oxygen). In this model, we adopt a high (but not total) freeze out efficiency. In Fig. 1 the Model 3 fractional abundances of selected species versus the logarithmic age of the clump, are plotted. The species selected for inclusion in Fig. 1 correspond to species for which SWAS gave constraints $\left(\mathrm{H}_{2} \mathrm{O}\right.$ and $\left.\mathrm{O}_{2}\right)$ and species observed previously (see later). In the models considered, $X\left(\mathrm{O}_{2}\right)$ is $<10^{-6}$ both at early times $(<1 \mathrm{Myr})$ (because of its slow formation) and at late times ( $>1.7 \mathrm{Myr}$ in these models) where freeze-out on to grains is removing molecules from the gas. Between these two times there is a "peak" in both species. The duration of these three phases, which we will call "formation", "equilibrium" and "depletion" stages, are model dependent. Water follows a similar behaviour to that of $\mathrm{O}_{2}$ although $X\left(\mathrm{O}_{2}\right)$ often exceeds the limit placed by the SWAS observations. A higher freeze--out efficiency would provide a more efficient loss route for water; however, such a solution would limit the lifetime of the clump to less than 3 Myrs as otherwise almost all molecular species would be frozen out. As expected, an oxygen-poor gas favours lower abundances of water and molecular oxygen, but only by factors comparable to the change in total oxygen abundance. 
Table 1. Model parameters: number densities are in $\mathrm{cm}^{-3}$; the gas temperature is in Kelvin; see text for a full explanation of the nomenclature

\begin{tabular}{|c|c|c|c|c|c|c|c|c|}
\hline & $n_{\mathrm{H}_{2}}$ & $T_{\text {gas }}$ & $F R$ & $A_{V}$ & & $X(\mathrm{O})$ & $\begin{array}{c}\mathrm{CO}-\mathrm{N}_{2} \\
\text { freeze-out }\end{array}$ & $\begin{array}{c}\mathrm{H}_{3} \mathrm{O}^{+} \\
\text {branching ratio }\end{array}$ \\
\hline 1 & $10^{4}$ & 10 & 0 & 15 & $\mathrm{nc}$ & $\mathrm{s}$ & yes & VC97 \\
\hline 2 & $10^{4}$ & 10 & 0.3 & 15 & $\mathrm{nc}$ & $\mathrm{S}$ & yes & VC97 \\
\hline 3 & $10^{4}$ & 10 & 0.7 & 15 & nc & ns & yes & VC97 \\
\hline 4 & $10^{4}$ & 10 & 0.3 & 15 & $\mathrm{nc}$ & ns & yes & VC97 \\
\hline 5 & $10^{4}$ & 10 & 0.3 & 2 & c & $\mathrm{S}$ & yes & VC97 \\
\hline 6 & $10^{4}$ & 10 & 0.3 & 5 & $\mathrm{c}$ & $\mathrm{S}$ & yes & VC97 \\
\hline 7 & $10^{4}$ & 10 & 1 & 5 & $\mathrm{c}$ & $\mathrm{s}$ & yes & VC97 \\
\hline 8 & $10^{4}$ & 10 & 0.3 & 15 & $\mathrm{c}$ & ns & yes & VC97 \\
\hline 9 & $10^{4}$ & 10 & 0.3 & 15 & c & ns & no & VC $97^{*}$ \\
\hline 10 & $10^{4}$ & 10 & 0.3 & 15 & $\mathrm{c}$ & ns & no & VC97 \\
\hline 11 & $10^{4}$ & 10 & 0.3 & 15 & $\mathrm{c}$ & ns & yes & W96 \\
\hline 12 & $10^{4}$ & 10 & 0.3 & 15 & $\mathrm{c}$ & $\mathrm{s}$ & yes & VC97 \\
\hline 13 & $10^{4}$ & 10 & 0.3 & 15 & $\mathrm{c}$ & $\mathrm{s}$ & yes & W96 \\
\hline 14 & $10^{4}$ & 10 & 0.3 & 2 & $\mathrm{nc}$ & $\mathrm{s}$ & yes & VC97 \\
\hline 15 & $10^{4}$ & 10 & 0.3 & 5 & $\mathrm{nc}$ & $\mathrm{s}$ & yes & VC97 \\
\hline 16 & $10^{4}$ & 10 & 0.3 & 5 & $\mathrm{c}$ & ns & yes & VC97 \\
\hline 17 & $10^{4}$ & 10 & 0.7 & 15 & nc & ns & no & VC97 \\
\hline 18 & $10^{4}$ & 10 & 0.7 & 15 & nc & ns & yes & W96 \\
\hline 19 & $10^{4}$ & 10 & 0.7 & 15 & $\mathrm{nc}$ & $\mathrm{s}$ & yes & VC97 \\
\hline 20 & $10^{4}$ & 10 & 0.7 & 15 & nc & $\mathrm{s}$ & yes & W96 \\
\hline 21 & $10^{4}$ & 10 & 0 & 15 & nc & ns & yes & VC97 \\
\hline 22 & $10^{4}$ & 10 & 0 & 2 & $\mathrm{c}$ & $\mathrm{s}$ & yes & VC97 \\
\hline 23 & $10^{4}$ & 10 & 1 & 15 & $\mathrm{c}$ & ns & yes & VC97 \\
\hline 24 & $10^{4}$ & 10 & 1 & 15 & $\mathrm{c}$ & $\mathrm{s}$ & yes & VC97 \\
\hline 25 & $10^{4}$ & 10 & 1 & 2 & $\mathrm{nc}$ & $\mathrm{s}$ & yes & VC97 \\
\hline 26 & $10^{4}$ & 10 & 1 & 2 & $\mathrm{c}$ & $\mathrm{S}$ & yes & VC97 \\
\hline 27 & $10^{4}$ & 10 & 1 & 5 & nc & $\mathrm{s}$ & yes & VC97 \\
\hline 28 & $10^{4}$ & 10 & 1 & 5 & $\mathrm{c}$ & ns & yes & VC97 \\
\hline 29 & $10^{4}$ & 30 & 0.3 & 2 & $\mathrm{nc}$ & $\mathrm{s}$ & yes & VC97 \\
\hline 30 & $10^{4}$ & 30 & 0.3 & 5 & nc & $\mathrm{s}$ & yes & VC97 \\
\hline 31 & $10^{4}$ & 30 & 1 & 15 & $\mathrm{c}$ & ns & no & VC97 \\
\hline 32 & $10^{4}$ & 30 & 1 & 2 & nc & $\mathrm{s}$ & yes & VC97 \\
\hline 33 & $10^{4}$ & 30 & 1 & 5 & nc & $\mathrm{S}$ & yes & VC97 \\
\hline 34 & $10^{5}$ & 10 & 0.3 & 2 & nc & $\mathrm{s}$ & yes & VC97 \\
\hline 35 & $10^{5}$ & 10 & 0.3 & 2 & $\mathrm{c}$ & $\mathrm{s}$ & yes & VC97 \\
\hline 36 & $10^{5}$ & 10 & 0.3 & 5 & nc & $\mathrm{S}$ & yes & VC97 \\
\hline 37 & $10^{5}$ & 10 & 0.3 & 5 & $\mathrm{c}$ & $\mathrm{s}$ & yes & VC97 \\
\hline 38 & $10^{5}$ & 10 & 0.7 & 15 & nc & ns & no & VC97 \\
\hline 39 & $10^{5}$ & 10 & 1 & 15 & $\mathrm{c}$ & $\mathrm{s}$ & no & VC97 \\
\hline 40 & $10^{5}$ & 10 & 1 & 2 & nc & $\mathrm{s}$ & yes & VC97 \\
\hline 41 & $10^{5}$ & 10 & 1 & 2 & c & $\mathrm{S}$ & yes & VC97 \\
\hline 42 & $10^{5}$ & 10 & 1 & 5 & $\mathrm{nc}$ & $\mathrm{s}$ & yes & VC97 \\
\hline 43 & $10^{5}$ & 10 & 1 & 5 & $\mathrm{c}$ & $\mathrm{s}$ & yes & VC97 \\
\hline 44 & $10^{5}$ & 30 & 0.3 & 15 & $\mathrm{c}$ & ns & yes & VC97 \\
\hline 45 & $10^{5}$ & 30 & 0.3 & 15 & c & $\mathrm{s}$ & yes & VC97 \\
\hline 46 & $10^{5}$ & 30 & 0.3 & 2 & nc & $\mathrm{s}$ & yes & VC97 \\
\hline 47 & $10^{5}$ & 30 & 0.3 & 2 & $\mathrm{c}$ & $\mathrm{s}$ & yes & VC97 \\
\hline 48 & $10^{5}$ & 30 & 0.3 & 30 & $\mathrm{c}$ & ns & yes & VC97 \\
\hline 49 & $10^{5}$ & 30 & 0.3 & 30 & c & $\mathrm{s}$ & yes & VC97 \\
\hline 50 & $10^{5}$ & 30 & 0.3 & 5 & nc & $\mathrm{S}$ & yes & VC97 \\
\hline 51 & $10^{5}$ & 30 & 0.3 & 5 & $\mathrm{c}$ & $\mathrm{S}$ & yes & VC97 \\
\hline 52 & $10^{5}$ & 30 & 1 & 15 & $\mathrm{c}$ & ns & yes & VC97 \\
\hline 53 & $10^{5}$ & 30 & 1 & 15 & c & ns & no & VC96 \\
\hline
\end{tabular}


Table 1. continued

\begin{tabular}{|c|cccccccc|}
\hline & $n_{\mathrm{H}_{2}}$ & $T_{\text {gas }}$ & $F R$ & $A_{V}$ & & $X(\mathrm{O})$ & $\begin{array}{c}\mathrm{CO}-\mathrm{N}_{2} \\
\text { freeze-out }\end{array}$ & $\begin{array}{c}\mathrm{H}_{3} \mathrm{O}^{+} \\
\text {branching ratio }\end{array}$ \\
\hline 54 & $10^{5}$ & 30 & 1 & 15 & $\mathrm{c}$ & $\mathrm{s}$ & yes & VC97 \\
55 & $10^{5}$ & 30 & 1 & 15 & $\mathrm{c}$ & $\mathrm{s}$ & yes & W96 \\
56 & $10^{5}$ & 30 & 1 & 2 & $\mathrm{nc}$ & $\mathrm{s}$ & yes & VC97 \\
57 & $10^{5}$ & 30 & 1 & 2 & $\mathrm{c}$ & $\mathrm{s}$ & yes & VC97 \\
58 & $10^{5}$ & 30 & 1 & 30 & $\mathrm{c}$ & $\mathrm{s}$ & yes & VC97 \\
59 & $10^{5}$ & 30 & 1 & 5 & $\mathrm{nc}$ & $\mathrm{s}$ & yes & VC97 \\
60 & $10^{5}$ & 30 & 1 & 5 & $\mathrm{c}$ & $\mathrm{s}$ & yes & VC97 \\
61 & $10^{6}$ & 30 & 0.3 & 15 & $\mathrm{c}$ & $\mathrm{ns}$ & yes & VC97 \\
62 & $10^{6}$ & 30 & 0.3 & 15 & $\mathrm{c}$ & $\mathrm{s}$ & yes & VC97 \\
63 & $10^{6}$ & 30 & 0.3 & 30 & $\mathrm{c}$ & $\mathrm{ns}$ & yes & VC97 \\
64 & $10^{6}$ & 30 & 0.3 & 30 & $\mathrm{c}$ & $\mathrm{s}$ & yes & VC97 \\
65 & $10^{6}$ & 30 & 1 & 15 & $\mathrm{c}$ & $\mathrm{ns}$ & yes & VC97 \\
66 & $10^{6}$ & 30 & 1 & 15 & $\mathrm{c}$ & $\mathrm{s}$ & yes & VC97 \\
67 & $10^{6}$ & 30 & 1 & 30 & $\mathrm{c}$ & $\mathrm{s}$ & yes & VC97 \\
\hline \hline
\end{tabular}

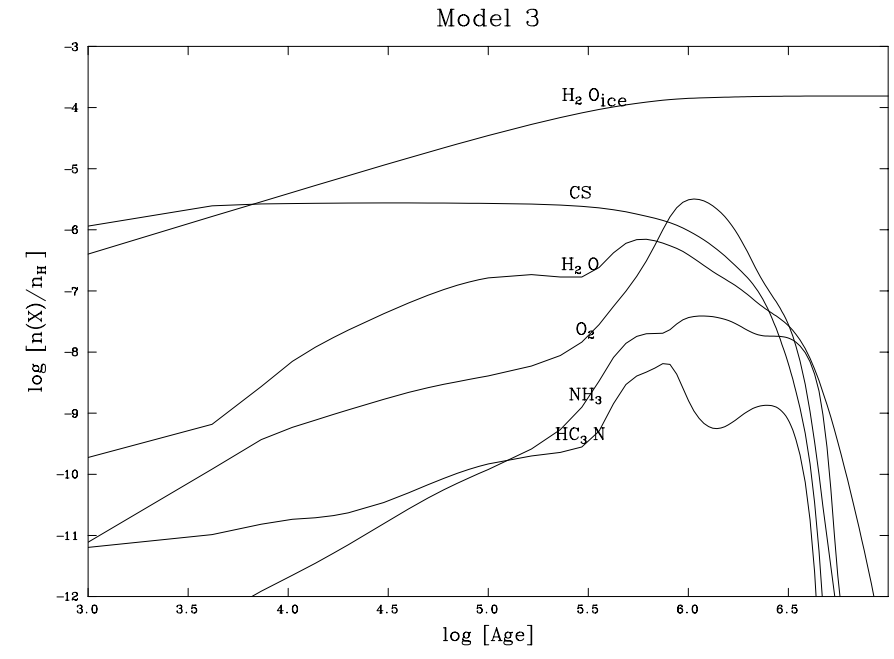

Fig. 1. Fractional abundances (relative to hydrogen) of selected species versus time (log) Model 3

Table 2. Fractional observed abundances, at $A_{V}=15 \mathrm{mag}$, with respect to hydrogen for selected species derived for the column densities of Takano et al. (1998), Hirahara et al. (1992). All the fractional abundances are derived for $A_{V}=15 \mathrm{mag}$

\begin{tabular}{|c|c|}
\hline & Species \\
\hline $\mathrm{HC}_{3} \mathrm{~N}$ & $2.4 \quad 10^{-9}$ \\
$\mathrm{NH}_{3}$ & $1.7 \quad 10^{-8}$ \\
$\mathrm{CS}$ & $1.8 \quad 10^{-10}$ \\
\hline
\end{tabular}

Also plotted in Fig. 1 are a few selected species detected in core B of TMC-1 (same line of sights as SWAS). Table 2 lists their observed fractional abundances: the $\mathrm{HC}_{3} \mathrm{~N}$ and $\mathrm{NH}_{3}$ have been calculated from the column densities derived by Takano et al. (1998) at the $\mathrm{NH}_{3}$ peak (core B) and CS from the column density observed by Hirahara et al. (1992). We also plot the fractional abundance of $\mathrm{H}_{2} \mathrm{O}_{\text {ice }}$ which is of course entirely created by grain surface chemistry when freeze out is included.

None of the models reproduces the observed abundances of these species during the "formation" stage when
$\mathrm{O}_{2}$ and $\mathrm{H}_{2} \mathrm{O}$ are low. However during the "freeze-out" stage, before everything is depleted, both $\mathrm{HC}_{3} \mathrm{~N}$ and $\mathrm{NH}_{3}$ are close to their observational values. CS would also be close to its observational value if we depleted it by a factor of 100: it should be noted that the abundances of sulphurbearing species shown in this paper are overestimated because the solar abundance of sulphur has been used in these calculations. Perhaps the actual value should be reduced by a factor of about 100 (e.g. Viti \& Williams 1999). However, as the mechanism for sulphur depletion is still unknown (e.g. Ruffle et al. 1999) we have assumed sulphur to have the solar elemental abundance rather than specify an uncertain parameter.

Abundances close to the observed values appear in the "depletion" phase. $\mathrm{HC}_{3} \mathrm{~N}$ in particular increases significantly when depletion is effective (cf. Ruffle et al. 1997). Gwenlan et al. (2000) reached a similar conclusion when discussing the chemistry of $\mathrm{HC}_{3} \mathrm{~N}$ for TMC- 1 . We have not attempted here to match precisely the abundances of core B in TMC- -1 , but merely used them as indicative of the chemistry in dark quiescent clouds. We conclude that the general distribution of abundances in starless cores, including those measured in the SWAS observations, could be interpreted as a late-time chemistry in an object in which freeze-out of molecules on to dust is occurring. However, the consequence of this interpretation is that the object has a limited existence, of duration of about $3 \mathrm{My}$. The close match between our abundances and the SWAS observations is however limited to a very short epoch: it is unlikely that all the cores observed by SWAS are at the same age; in a later section, we therefore explore a modified solution where selective freeze out occurs and we show that this would significantly extend the period over which the abundances of observed species are well reproduced. In general, the implication is that there must be (in this interpretation) a cycle of material in the interstellar medium through stages such as those in the models, on this timescale. 


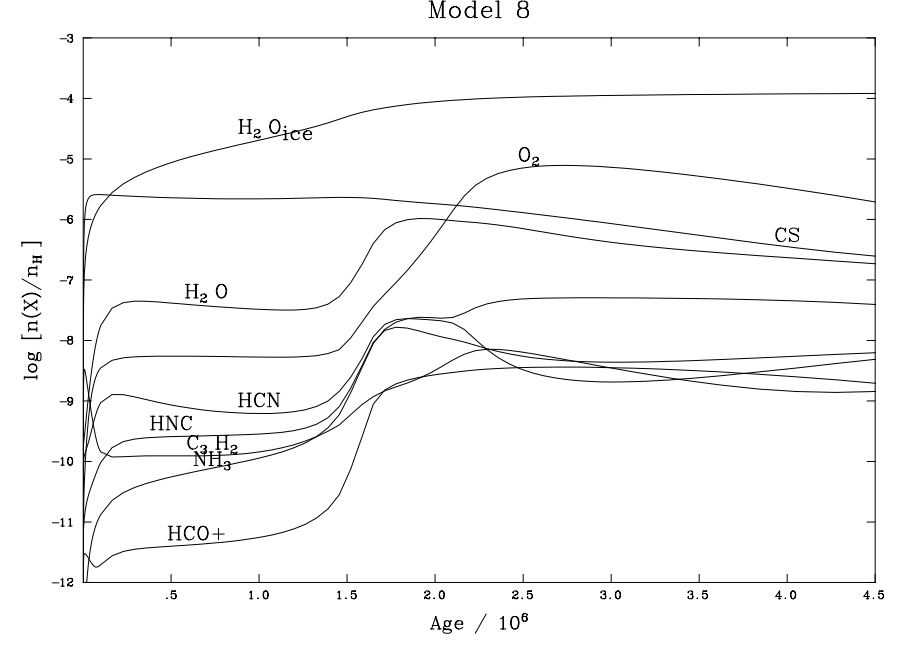

Fig. 2. Fractional abundance (relative to hydrogen nuclei) of selected species as a function of age for Model 8

\subsubsection{Low density gas}

Neufeld et al. (2000) reported observations with SWAS along the line of sight towards Sgr B2 $\left(n_{\mathrm{H}} \leq 10^{4} \mathrm{~cm}^{-3}\right.$, $\left.T_{\text {gas }} \sim 10-30 \mathrm{~K}\right)$. This is a long, low-extinction path. They found that water vapour in the foreground gas has a high fractional abundance of $\sim 610^{-7}$, significantly higher than found in dense cores. A low fractional abundance of molecular oxygen, less than $10^{-6}$, was also found with SWAS on these lines of sight.

To represent material along this line of sight, we have concentrated our analysis on models of collapsing clouds, as star formation may be present along this line of sight. Model 8 is the model in which collapse is included and CO and $\mathrm{N}_{2}$ freeze-out occurs with chemical results agreeing most closely with those we consider appropriate for the Sgr B2 line of sight; Fig. 2 shows results for Model 8.

The high visual extinction in this model favours a higher abundance of water at early times because $\mathrm{H}_{2} \mathrm{O}$ photodissociation is impeded. Water and molecular oxygen are in harmony with the observed constraints for a brief period around 1.5 Myrs which is when the final density in the collapse is reached. After that brief period, the $\mathrm{H}_{2} \mathrm{O}$ and $\mathrm{O}_{2}$ abundances rise quickly to unacceptably high values. If the clump is initially oxygen-poor the formation of $\mathrm{O}_{2}$ is delayed relative to $\mathrm{H}_{2} \mathrm{O}$, probably because oxygen is more easily locked into water than molecular oxygen so a decrease in initial oxygen will delay the formation of $\mathrm{O}_{2}$.

In the foreground gas along the line of sight of Sgr B2, many species have been observed. We have chosen to compare our models to some of the observational results of $\mathrm{NH}_{3}$ (Hüttemeister et al. 1993), CS and $\mathrm{C}_{3} \mathrm{H}_{2}$ (Greaves et al. 1992), and $\mathrm{HCO}^{+}, \mathrm{HCN}$ and $\mathrm{HNC}$ (Linke et al. 1981) (see Table 3). Note that while the column densities for $\mathrm{NH}_{3}$, CS and $\mathrm{C}_{3} \mathrm{H}_{2}$ are derived from observations of cool components detected at $\sim-40 \mathrm{~km} \mathrm{~s}^{-1}$ the column densities of $\mathrm{HCO}^{+}, \mathrm{HCN}$ and $\mathrm{HNC}$ are determined from absorption depths in the $\sim 129 \mathrm{~km} \mathrm{~s}^{-1}$ feature of Sgr B2.
Table 3. Observed fractional abundances with respect to molecular hydrogen for selected species. All are derived for an assumed $A_{V}$ of $15 \mathrm{mag}$, and a standard $A_{V}$ to hydrogen nuclei ratio

\begin{tabular}{|c|c|}
\hline & Species \\
\hline $\mathrm{NH}_{3}$ & $310^{-8}$ \\
$\mathrm{CS}$ & $1.210^{-9}$ \\
$\mathrm{C}_{3} \mathrm{H}_{2}$ & $1.110^{-10}$ \\
$\mathrm{HCN}$ & $1.110^{-9}$ \\
$\mathrm{HNC}^{+}$ & $2.610^{-9}$ \\
$\mathrm{HCO}^{+}$ & $8.7510^{-10}$ \\
\hline
\end{tabular}

This should not be a problem as although SWAS observations along the line of sight of Sgr B2 have distinguished various regions kinematically (Neufeld et al. 2000), the water abundances derived was averaged along the line of sight.

In the region where the Model 8 water and molecular oxygen agree with observations (between 1.5 and 2.1 million years), results for $\mathrm{NH}_{3}$ and $\mathrm{HCO}^{+}$are in reasonable agreement with observations, $\mathrm{HCN}$ and $\mathrm{HNC}$ are slightly overabundant while CS (depleted by 100) and $\mathrm{C}_{3} \mathrm{H}_{2}$ are overabundant by about one half to 1 order of magnitude. As is the case for cores with little star formation (Sect. 2.2.1), the chemistry of low density gas is very timedependent. The relatively high $\mathrm{H}_{2} \mathrm{O}$ abundance together with the non-detection of $\mathrm{O}_{2}$ provides a severe constraint which can only be met for rather short periods within the scenario described here. High visual extinction and freeze-out lead to better reproduction of the observed abundances, although only for short periods of time. If a typical clump survives for $10 \mathrm{Myr}$ it is unlikely that we would observe the same abundances towards different lines of sight. The short epoch during which model results are compatible with observational results could imply that the clumps are much shorter lived than $10 \mathrm{Myr}$; perhaps, they are dispersed by stellar outflows or depletion occurs rapidly enough that a clump quickly becomes undetectable.

\subsubsection{High-mass star-forming regions}

These regions are characterized by the following physical properties: $n_{\mathrm{H}} \geq 10^{5} \mathrm{~cm}^{-3} ; T_{\text {gas }}=20-40 \mathrm{~K} ; A_{V}>$ 10 mag. The results from SWAS show that in these regions the water fractional abundance is very low, $10^{-9}<$ $X\left(\mathrm{H}_{2} \mathrm{O}\right)<$ few $10^{-8}$ (Bergin et al. 2000), and the upper limit on $X\left(\mathrm{O}_{2}\right)$ is $10^{-6}$. We choose to represent this material with some of the high density models. Figure 3 shows the results for selected species from Model 52 which is the high density model with $\mathrm{CO}$ and $\mathrm{N}_{2}$ freeze-out giving the best agreement with water and molecular oxygen observations for a period of up to 1.5 million years. This timescale varies according to the initial and final 
Model 52

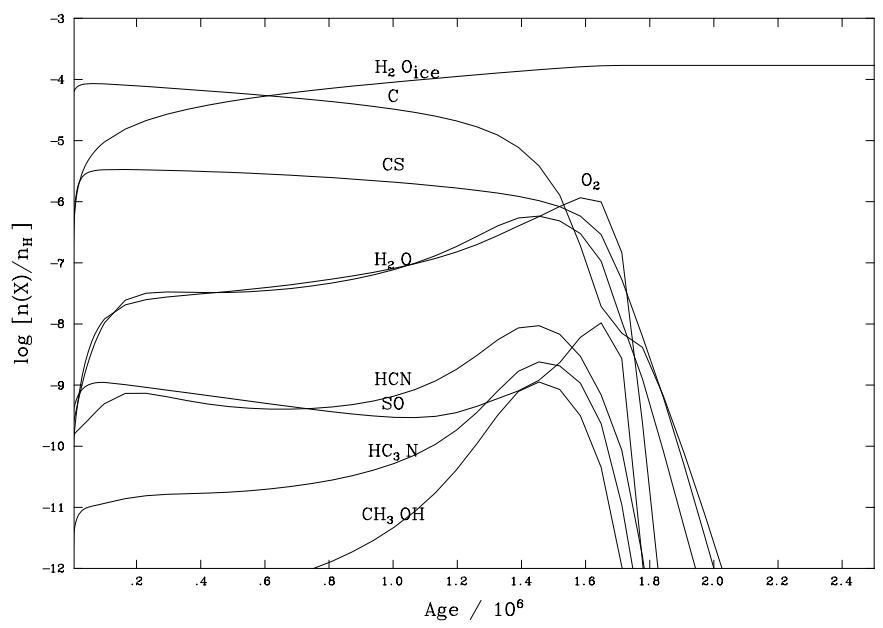

Fig. 3. Fractional abundances (relative to hydrogen nuclei) of selected species as a function of age for Model 52

Table 4. Fractional observed abundances with respect to hydrogen for selected species derived from fractional abundances relative to CO from Bergin et al. (1999). We adopted a column density for CO of $310^{18} \mathrm{~cm}^{-2}$ (from core Cepheus in Bergin et al.) and we estimated its fractional abundance with respect to hydrogen assuming a visual extinction of $\sim 15 \mathrm{mag}$

\begin{tabular}{|c|c|}
\hline & Species \\
\hline $\mathrm{CS}$ & $410^{-9}$ \\
$\mathrm{CH}_{3} \mathrm{OH}$ & $210^{-8}$ \\
$\mathrm{HC}_{3} \mathrm{~N}$ & $210^{-10}$ \\
$\mathrm{SO}$ & $410^{-9}$ \\
$\mathrm{HCN}$ & $1.210^{-8}$ \\
$\mathrm{C}$ & $610^{-6}-210^{-5}$ \\
\hline
\end{tabular}

densities employed as it is a function of the gas-phase species depletion on the grains.

We note however that none of models reproduce the low water abundance observed with SWAS until freeze-out begins to dominate. High visual extinction and a final density of $10^{5} \mathrm{~cm}^{-3}$ are favoured.

Bergin et al. (1997) presented results of an observational study covering three giant cloud cores. We use their observational results to compare with our model results for some species (see Table 4 for a list of the species selected with their observational values). We find that there is only a very small period of time during which most of the measured abundances are matched by our models; the best match is reached at $t \sim 1.5$ Myrs, where HCN, CS, $\mathrm{C}$ and $\mathrm{HC}_{3} \mathrm{~N}$ model abundances are close to the observational values, although $\mathrm{CH}_{3} \mathrm{OH}$ and $\mathrm{SO}$ (depleted by some depletion factor) appear to be underabundant.

Regions of high density are chemically and dynamically very complex because of the effects of outflows and shocks that excite or heat the gas and the grains: some grain evaporation might occur leading to chemical differentiation due to the different binding energies of the frozen species. The simple studies presented here do not take into account such effects, and cannot be expected to account in any detail for averaged measurements represented by the SWAS results. Nevertheless, one can infer that if the basic chemistry is correct, then for the models to be valid it means that the timescales in high-mass star-forming regions must be very short.

\subsection{Selective freeze-out}

In all the cases considered so far, it has been assumed that the nature of the gas-grain interaction is the same for all species other than $\mathrm{H}_{2}$ and He, i.e every species colliding with a grain sticks and is retained. Therefore, the sticking probability has been assumed to be the same for all species (except $\mathrm{H}_{2}$ and $\mathrm{He}$ ). Such assumptions may be unjustified, and there have been many studies of systems in which it has been assumed that certain molecules are more readily desorbed into the gas, and not retained on grain surfaces (Millar \& Nejad 1985; Hartquist \& Williams 1990; Willacy \& Williams 1993; Willacy et al. 1994; Gwenlan et al. 2000). The effect of retaining weakly bound molecules such as $\mathrm{CO}$ and $\mathrm{N}_{2}$ in the gas phase is to prolong the epoch of gas-phase chemistry in molecular clouds; the carbon and oxygen in $\mathrm{CO}$, for example, is re-cycled through hydrocarbons and water and these molecules ultimately freeze-out permanently. In particular, the $\mathrm{C}: \mathrm{O}$ ratio in the chemistry is changed during this phase, and the results are of a chemistry in which $[\mathrm{C}] /[\mathrm{O}]$ is close to unity (similar to the cyclic models of Nejad \& Williams 1992).

For models 10, 17, 53, 31, 38 (see Table 1) we assumed that both $\mathrm{CO}$ and $\mathrm{N}_{2}$ are rapidly desorbed, so that the atoms in those molecules feed a late chemistry. Results for the abundances of some species are given in Fig. 4 for Models 10, 17, 31 and 53 .

Model 17 may be compared directly to Model 3 (cf. Fig. 1), which we have used to represent starless cores. The consequence of the selective freeze-out which maintains $\mathrm{CO}$ and $\mathrm{N}_{2}$ in the gas is that the period over which the chemistry of observed species, such as $\mathrm{H}_{2} \mathrm{CO}, \mathrm{NH}_{3}$ and $\mathrm{HC}_{3} \mathrm{~N}$, is reproduced reasonably well by the models is extended considerably from about $1 \mathrm{My}$ to several Myrs. The Model $17 \mathrm{H}_{2} \mathrm{O}$ and $\mathrm{O}_{2}$ abundances satisfy the observed constraints for ages greater than $\sim 2$ My (cf. Fig. 4). If we increase the density of our clump (Model 38) the $\mathrm{H}_{2} \mathrm{O}$ and $\mathrm{O}_{2}$ observational constraints are satisfied already by $0.5 \mathrm{My}$.

Model 10 may be compared directly to Model 8 (see Fig. 2). The model water fractional abundance rises to near $10^{-6}$, but the $\mathrm{O}_{2}$ fractional abundance exceeds the SWAS limit by a factor of about 3 for at least part of the chemical evolution. This demonstrates how closely the $\mathrm{H}_{2} \mathrm{O}$ and $\mathrm{O}_{2}$ chemistries are linked. However, in warm (mainly neutral) regions behind shocks the formation of $\mathrm{H}_{2} \mathrm{O}$ is rapid while $\mathrm{O}_{2}$ remains a minor channel and it is in fact destroyed in strong shocks. Hence, it is quite possible that the relatively high water abundance observed towards the Galactic Centre, $X\left(\mathrm{H}_{2} \mathrm{O}\right) \sim 10^{-6}$, could be 

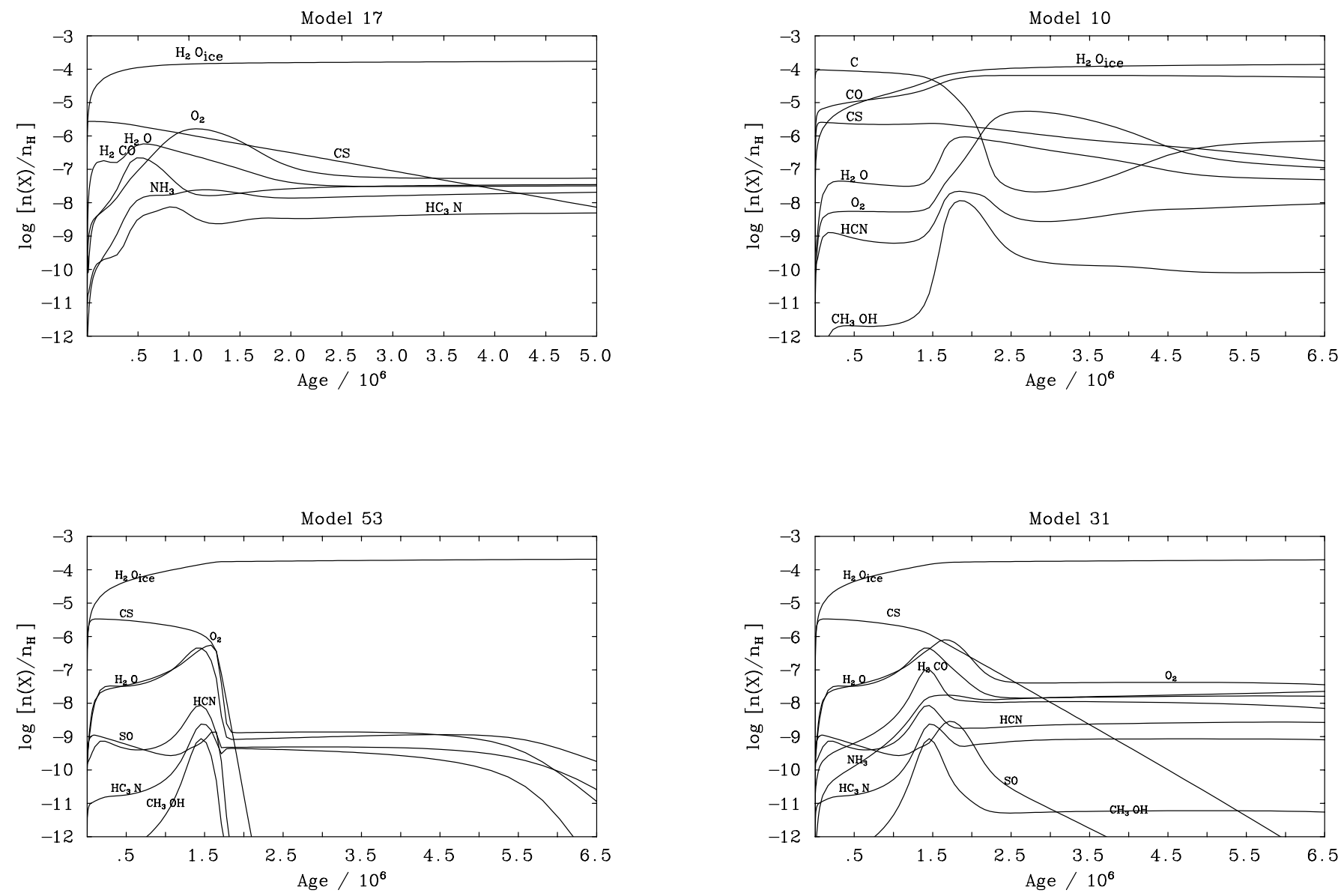

Fig. 4. Fractional abundances (relative to hydrogen) of selected species versus time for models where $\mathrm{CO}$ and $\mathrm{N}_{2}$ are immediately released back into the gas after freezing-out

the result of one or more shocks along this line of sight. This line of sight certainly passes through several starforming regions where shocks may be expected to occur, and in which the $\mathrm{H}_{2} \mathrm{O}: \mathrm{O}_{2}$ balance is strongly increased. In warm post-shock gas, $X\left(\mathrm{H}_{2} \mathrm{O}\right) \sim 10^{-4}$, and therefore the fraction of the path towards the Galactic Centre that would be required by the detection of $\mathrm{H}_{2} \mathrm{O}$ to be shocked would be around $1 \%$, corresponding to a column density of warm $\mathrm{H}_{2}$ of $\sim 10^{21} \mathrm{~cm}^{-2}$.

Model 53 can be compared directly with Model 52 (see Fig. 3). The consequent chemistry has a much extended duration in the case of Model 53 to about 6 My (Fig. 4). However, the freeze-out of CS (which is largely unaffected by this late-phase chemistry) is so rapid at these high densities that it would be undetectable at the levels shown in this model. We have, therefore, examined a very similar case to Model 53, but for a lower density gas (Model 31). Note however that a density of $10^{4} \mathrm{~cm}^{-3}$ may not be representative of many high mass star forming regions. Model 31 shows that from about $2 \mathrm{My}$ onwards the $\mathrm{H}_{2} \mathrm{O}$ and $\mathrm{O}_{2}$ abundances satisfy the SWAS constraints, while other species such as $\mathrm{H}_{2} \mathrm{CO}, \mathrm{HCN}, \mathrm{NH}_{3}$, CS and $\mathrm{HCN}$ have levels that are generally around the levels detected; SO and $\mathrm{CH}_{3} \mathrm{OH}$ however still remain too low, and this suggests that their chemistries are associated with processes not included in these models.

\subsection{The branching ratios for $\mathrm{H}_{3} \mathrm{O}^{+}$electronic recombination}

We have studied the effect of varying the branching ratios for $\mathrm{H}_{3} \mathrm{O}^{+}$dissociative recombination with electrons. We have chosen one model per scenario and we have recomputed them with the Williams et al. (1996) branching ratios for the dissociative recombination of $\mathrm{H}_{3} \mathrm{O}^{+}$with electrons. The models computed with the Williams et al. (1996) branching ratios are models 11, 13, 18 and 20.

The effect of lowering so dramatically the branching ratio for the main formation channel for water is, as expected, to reduce water abundance for all the three scenarios: for cores with little star formation, this constitutes an improvement as we require water to be very low. However, for low density gas such as along the line of sight of Sgr B2, water is observed to be quite abundant and therefore the SWAS requirements are more poorly matched by this model. For high mass star forming regions a decrease in water formation is desired so models with a low branching ratio are well suited. However, we also obtain a decrease in methanol and $\mathrm{SO}$ which is not desired; 

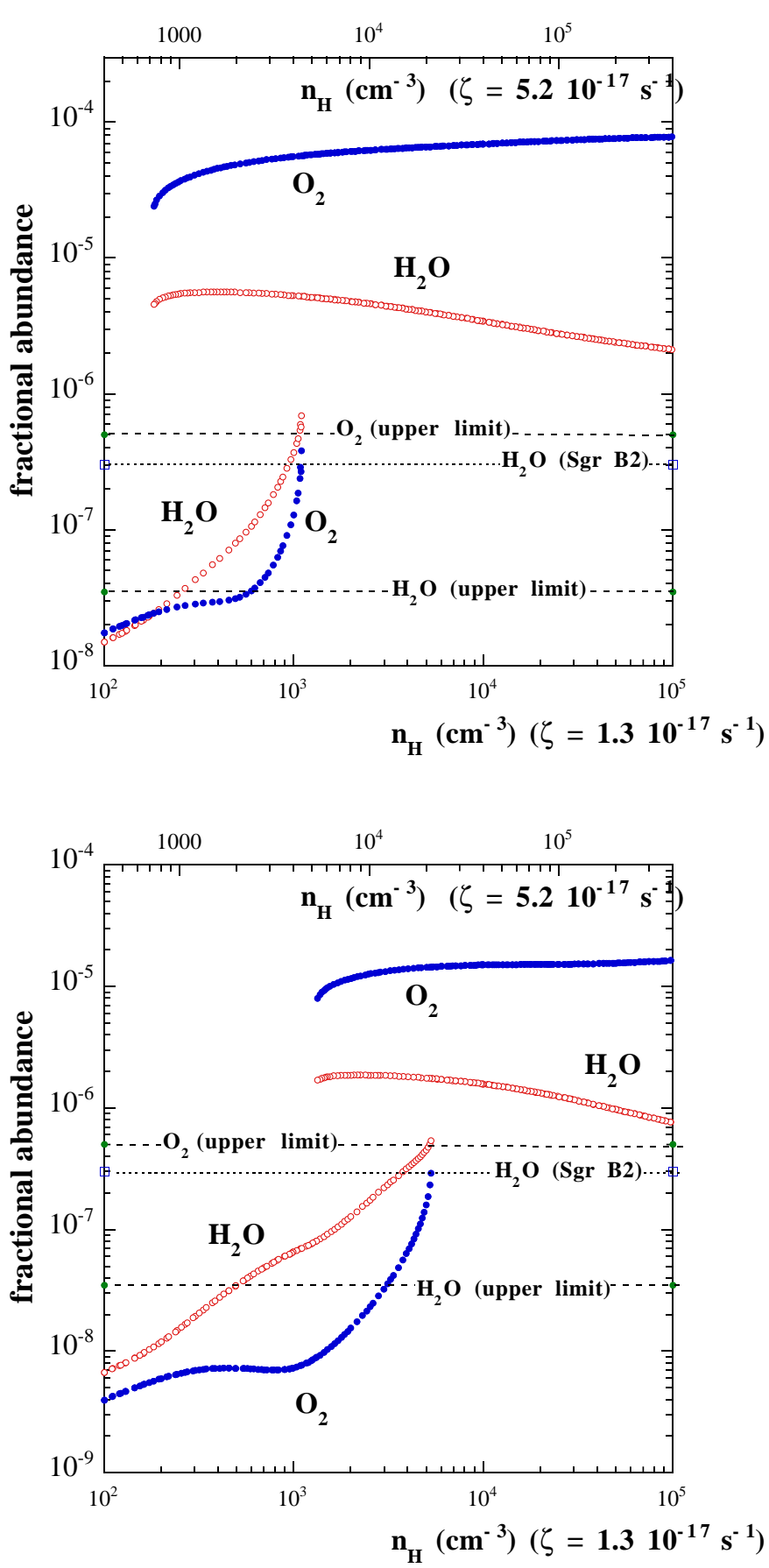

Fig. 5. Steady state stable solutions for a range of densities between 100 and $10^{5} \mathrm{~cm}^{-3}$ when $\zeta=1.310^{-17} \mathrm{~s}^{-1}$ (lower $x$ axis) and for a range of densities between 400 and $410^{5} \mathrm{~cm}^{-3}$ when $\zeta=5.210^{-17} \mathrm{~s}^{-1}$ (upper $x$ axis). a) standard elemental depletion of oxygen; b) non standard elemental depletion of oxygen. The dotted horizontal lines correspond to the observed upper limit for $\mathrm{O}_{2}$ and $\mathrm{H}_{2} \mathrm{O}$. The short-dashed line corresponds to the observed fractional abundance of water towards Sgr B2

however, as noted above, these species are not well described in these models. Very recently, new measurements of the $\mathrm{H}_{3} \mathrm{O}^{+}$branching ratios by Jensen et al. (2000) have been published. Their measurements yield a higher branching ratio for $\mathrm{OH}$ formation and a lower branching ratio for $\mathrm{H}_{2} \mathrm{O}$ formation but both agree within a factor of 1.2 with the Vejby-Christensen et al. (1997) measurements.

Although the use of different branching ratios of $\mathrm{H}_{3} \mathrm{O}^{+}$ reduces the production of water and $\mathrm{O}_{2}$ dramatically, it does not alter the general chemical behaviour of the clump and therefore our previous discussions are not affected.

\section{Steady state chemical models and bistability}

\subsection{General considerations}

The same gas phase chemical network has been used for determining the steady state solutions of the atomic and molecular species in the gas phase. This is performed by solving $\mathrm{d} \boldsymbol{X} / \mathrm{d} t=0$ where $\boldsymbol{X}$ is a vector representing the abundances of all the gas phase species considered in the chemical network. No surface reaction, except for the formation of $\mathrm{H}_{2}$, has been introduced. Le Bourlot et al. (1993) have shown that the intrinsic non linearities present in the chemical equations may lead to a non linear behaviour of the phase space of the chemical solutions. For a given value of the control parameters (density, temperature, cosmic ionization rate, elemental abundances, etc.), the steady state solutions depend on the initial conditions, (on the atomic, ionic and molecular fractional abundances of oxygen, carbon, sulphur etc.). Then, two stable and one unstable steady state solutions can appear for a given set of control parameters, corresponding to an identical physical state of the gas. This behaviour is known as bistability and is a well known property of open thermodynamical systems. The two stable states have very different chemical properties. The Low Ionization Phase (LIP) solution corresponds to the standard solution found in most chemical models where the fractional ionization is low $\left(10^{-8}-10^{-7}\right)$ and is provided mainly by molecular and metallic ions. On the other hand, the High Ionization Phase (HIP) solution involves a larger fractional ionization $\left(10^{-5}-10^{-6}\right)$ dominated by atomic ions $\left(\mathrm{H}^{+}, \mathrm{C}^{+}\right.$, etc. $)$. Comparison between the LIP solutions and the time-dependent results obtained in the previous section (in the $F R=0$ model), integrated to late times leads to identical results, within the numerical accuracy. In fact, the steady state solutions depend only on the $n_{\mathrm{H}}$ to $\zeta$ ratio (Lee et al. 1998). So, we may display the stable steady state solutions for two different values of the cosmic ionization rate on the lower and upper abscissae axis by simply scaling the proton density abscissae axis by the same factor as the cosmic ionization rate if the temperature is assumed to be identical. Figure 5 shows the stable steady state solutions found for molecular oxygen and water within a range of densities between $10^{2}$ and $10^{5} \mathrm{~cm}^{-3}$ with a fixed temperature of $10 \mathrm{~K}$ for a cosmic ionization rate of $1.310^{-17} \mathrm{~s}^{-1}$ on the bottom $x$ axis whereas the same values correspond to a range of densities comprised between 400 and $410^{5} \mathrm{~cm}^{-3}$ for a cosmic ionization rate that is four times larger, as shown on the upper $x$ axis. Figure 5a displays the results obtained with the standard depletion of oxygen whereas 
Fig. 5b refers to the non standard depletion of oxygen (see previous section).

The two cases show a specific range of densities where bistability does occur. The LIP phase corresponds to the high density regions and leads to large fractional abundances of molecular oxygen and water. Such a result is in agreement with the various model results found in the literature (cf. Bergin et al. 2000). However, we find a range of densities where a high ionization phase is simultaneously present: the range is between 200 and $1000 \mathrm{~cm}^{-3}$ with the standard depletion of oxygen (respectively $800-4000 \mathrm{~cm}^{-3}$ for a cosmic ionization rate of $\left.5.210^{-17} \mathrm{~s}^{-1}\right)$ whereas it is shifted in the range $1300-5200 \mathrm{~cm}^{-3}$ (respectively $5200-$ $16000 \mathrm{~cm}^{-3}$ for a cosmic ionization rate of $5.210^{-17} \mathrm{~s}^{-1}$ ) with the non standard depletion of molecular oxygen. This latter situation offers a possible solution to the SWAS observations if the initial molecular state of the cloud (and the control parameters) are such that a HIP solution is obtained.

Since the beam of the SWAS satellite is as large as 4 arcminutes, it should also be kept in mind that the two chemical phases can be spanned in the same beam. Then, the resulting averaged fractional abundance could be a mixture of the two phases. It is, however, not possible to describe precisely the occurrence of such cases since various physical effects are neglected in these isothermal models, such as the possible coupling of the two phases via radiative transfer. Higher spatial resolution is then clearly required to derive more definitive conclusions. A preliminary suggestion of the presence of two chemical phases has indeed been found by Gerin et al. (1997) from observations of CS, SO and deuterated species in the high latitude cloud MCLD $123.5+24.9$ at much higher spatial resolution.

\subsection{Chemical discussion}

At this point, a chemical discussion on the formation and destruction processes of both molecular oxygen and water is interesting. Table 5 displays the main formation and destruction processes of $\mathrm{H}_{3}^{+}, \mathrm{O}_{2}$ and $\mathrm{H}_{2} \mathrm{O}$ which occur in both phases. In Table 5, we consider the solutions obtained for $n_{\mathrm{H}}=5000 \mathrm{~cm}^{-3}$ where both LIP and HIP solutions coexist, using the non standard value of oxygen depletion $\left(\mathrm{O} / n_{\mathrm{H}}=2.14\right)$.

A first conclusion of our analysis is that very few reactions, amongst the 4000 reactions involved in the chemical network, are really important for a given molecule.

\subsection{1. $\mathrm{H}_{3}+$}

$\mathrm{H}_{3}{ }^{+}$plays a key role in the chemistry of oxygen compounds. Indeed, $\mathrm{H}_{3}{ }^{+}+\mathrm{O}$ gives $\mathrm{OH}^{+}$which subsequently reacts with $\mathrm{H}_{2}$ giving $\mathrm{H}_{2} \mathrm{O}^{+}$. In dense cloud conditions $\mathrm{H}_{2} \mathrm{O}^{+}$reacts immediately with $\mathrm{H}_{2}$ giving $\mathrm{H}_{3} \mathrm{O}^{+}$. Then $\mathrm{H}_{3} \mathrm{O}^{+}$dissociatively recombines with electrons giving both $\mathrm{OH}$ and $\mathrm{H}_{2} \mathrm{O}$, as discussed in the previous section. The channel leading to $\mathrm{H}_{2} \mathrm{O}$ represents the entire gas-phase formation process for water whereas the subsequent reaction of $\mathrm{OH}$ with atomic oxygen gives molecular oxygen with a $100 \%$ contribution. The formation of $\mathrm{H}_{3}{ }^{+}$is only due to the reaction between $\mathrm{H}_{2}{ }^{+}$and $\mathrm{H}_{2}$. However $\mathrm{H}_{2}{ }^{+}$is formed from the impact of cosmic rays (CR) on molecular hydrogen through a direct photoionization process:

$\mathrm{H}_{2}+\mathrm{CR} \Rightarrow \mathrm{H}_{2}{ }^{+}+$electron with a ionization rate $\alpha \times \zeta$. There is another possible channel for this reaction involving dissociative ionization of $\mathrm{H}_{2}$ :

$\mathrm{H}_{2}+\mathrm{CR} \Rightarrow \mathrm{H}^{+}+\mathrm{H}+$ electron with a ionization rate $(1-\alpha) \times \zeta$.

The branching ratio between the two channels has been measured by Shah \& Gilbody (1982) for keV protons and the relevant astrophysical value has been calculated by Cravens \& Dalgarno (1975). The latter value of $\alpha$, 0.97 , is well within the range of measured values. We find it worthwhile to recall the difficulty of interpreting with standard models the low fractional abundance of $\mathrm{H}_{3}^{+}$detected in dense interstellar clouds by McCall et al. (1999). We have to keep in mind that cosmic rays are also critical for destruction of both $\mathrm{O}_{2}$ and $\mathrm{H}_{2} \mathrm{O}$. Indeed, the analysis reported in Table 5 involves secondary UV photons (generated by the excitation of $\mathrm{H}_{2}$ by secondary electrons produced after the interaction of cosmic rays and molecular hydrogen) which contribute significantly to the destruction of $\mathrm{O}_{2}$ and $\mathrm{H}_{2} \mathrm{O}$. The difficulties encountered in interpreting the SWAS data may then be linked to the $\mathrm{H}_{3}^{+}$ puzzle.

\subsubsection{The $\mathrm{O}+\mathrm{OH}$ reaction}

The $\mathrm{O}+\mathrm{OH}$ reaction is the dominant $\mathrm{O}_{2}$ formation process. Davidsson \& Stenholm (1990) predict, on the basis of an extended Langevin model for the calculation of the rate coefficient, that the reaction is rapid at very low temperatures. However, the reaction rate coefficient is not known experimentally at very low temperatures. Smith \& Stewart (1994) have found that the reaction proceeds rapidly for temperatures down to $158 \mathrm{~K}$. To extrapolate the value at low temperatures as is done in the present model is however highly uncertain. To this purpose, we have studied the possible influence of an activation barrier in the reaction rate coefficient. Figure 6 displays the fractional abundances of $\mathrm{O}_{2}$ and $\mathrm{H}_{2} \mathrm{O}$ for a proton density $n_{\mathrm{H}}$ of $10^{4} \mathrm{~cm}^{-3}$ with different values of the activation barrier. When the activation barrier is of the order of $80 \mathrm{~K}$, the fractional abundance of $\mathrm{O}_{2}$ drops to values lower than $10^{-6}$ whereas no sensible influence is found for the $\mathrm{H}_{2} \mathrm{O}$ fractional abundance. It is clearly highly desirable that new experiments or calculations address this reaction at very low temperatures. This would also affect the fractional abundance of molecular oxygen predicted in the models presented in Sect. 2. 
Table 5. Formation and destruction reactions relevant to $\mathrm{H}_{3}{ }^{+}, \mathrm{O}_{2}$ and $\mathrm{H}_{2} \mathrm{O}$

\begin{tabular}{|c|c|c|c|c|c|}
\hline Phase & & Formation reaction & percentage & Destruction reaction & percentage \\
\hline \multirow[t]{14}{*}{ LIP } & $\mathrm{H}_{3}{ }^{+}$ & $\mathrm{H}_{2}^{+}+\mathrm{H}_{2}$ & $100 \%$ & $\mathrm{H}_{3}{ }^{+}+\mathrm{CO}$ & $44 \%$ \\
\hline & & & & $\mathrm{H}_{3}^{+}+\mathrm{O}$ & $15 \%$ \\
\hline & & & & $\mathrm{H}_{3}^{+}+\mathrm{N}_{2}$ & $13 \%$ \\
\hline & & & & $\mathrm{H}_{3}{ }^{+}+$electron & $12 \%$ \\
\hline & $\mathrm{O}_{2}$ & $\mathrm{O}+\mathrm{OH}$ & $100 \%$ & $\mathrm{O}_{2}+$ secondary photons & $44 \%$ (dissociation) \\
\hline & & & & $\mathrm{O}_{2}+\mathrm{C}^{+}$ & $21 \%$ \\
\hline & & & & $\mathrm{O}_{2}+\mathrm{S}^{+}$ & $7 \%$ \\
\hline & & & & $\mathrm{O}_{2}+$ secondary photons & $7 \%$ (ionization) \\
\hline & & & & $\mathrm{O}_{2}+\mathrm{S}$ & $7 \%$ \\
\hline & & & & $\mathrm{O}_{2}+\mathrm{H}^{+}$ & $5 \%$ \\
\hline & $\mathrm{H}_{2} \mathrm{O}$ & $\mathrm{H}_{3} \mathrm{O}^{+}+$electron & $92 \%$ & $\mathrm{H}_{2} \mathrm{O}+\mathrm{H}_{3}^{+}$ & $47 \%$ \\
\hline & & $\mathrm{H}_{3} \mathrm{O}^{+}+\mathrm{CS}$ & $8 \%$ & $\mathrm{H}_{2} \mathrm{O}+\mathrm{HCO}^{+}$ & $17 \%$ \\
\hline & & & & $\mathrm{H}_{2} \mathrm{O}+$ secondary photons & $13 \%$ \\
\hline & & & & $\mathrm{H}_{2} \mathrm{O}+\mathrm{C}^{+}$ & $9 \%$ \\
\hline \multirow[t]{7}{*}{ HIP } & $\mathrm{H}_{3}{ }^{+}$ & $\mathrm{H}_{2}^{+}+\mathrm{H}_{2}$ & $100 \%$ & $\mathrm{H}_{3}{ }^{+}+$electron & $77 \%$ \\
\hline & & & & $\mathrm{H}_{3}{ }^{+}+\mathrm{CO}$ & $10 \%$ \\
\hline & & & & $\mathrm{H}_{3}^{+}+\mathrm{O}$ & $6 \%$ \\
\hline & $\mathrm{O}_{2}$ & $\mathrm{O}+\mathrm{OH}$ & $100 \%$ & $\mathrm{C}+\mathrm{O}_{2}$ & $82 \%$ \\
\hline & & & & $\mathrm{S}^{+}+\mathrm{O}_{2}$ & $8 \%$ \\
\hline & $\mathrm{H}_{2} \mathrm{O}$ & $\mathrm{H}_{3} \mathrm{O}^{+}+$electron & $100 \%$ & $\mathrm{H}_{2} \mathrm{O}+\mathrm{C}^{+}$ & $71 \%$ \\
\hline & & & & $\mathrm{H}_{2} \mathrm{O}+$ secondary photons & $11 \%$ \\
\hline
\end{tabular}

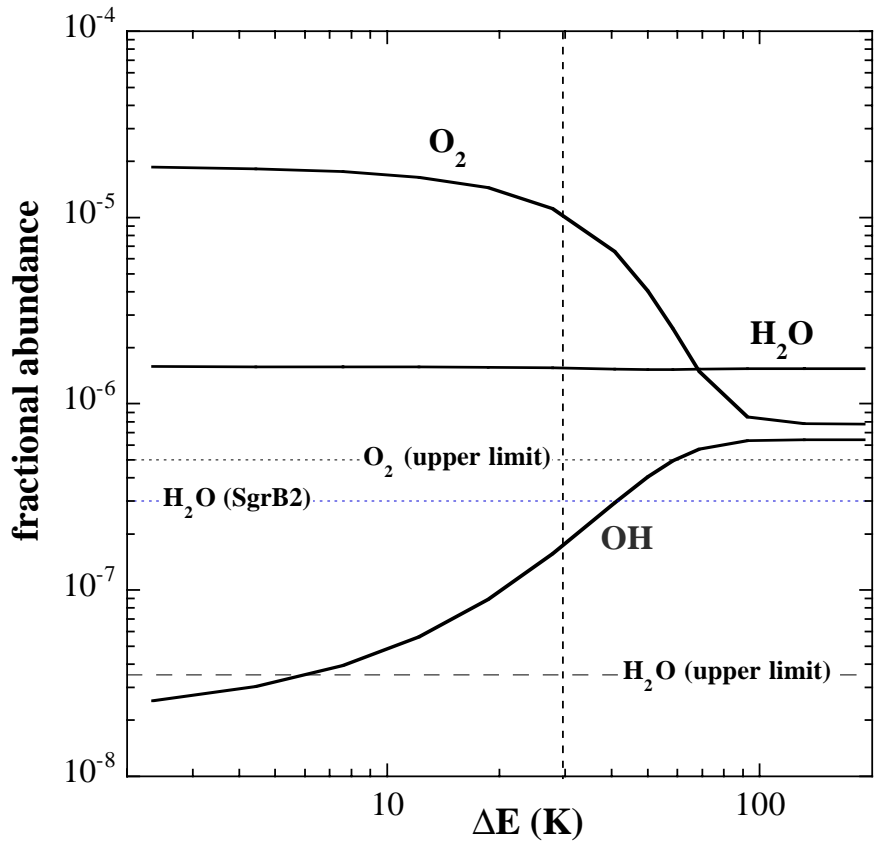

Fig. 6. Steady state fractional abundances of $\mathrm{O}_{2}, \mathrm{H}_{2} \mathrm{O}$ and $\mathrm{OH}$ for a proton density of $10^{4} \mathrm{~cm}^{-3}$ as a function of the activation barrier in the $\mathrm{O}+\mathrm{OH}$ reaction. The vertical line corresponds to the actual value used in the chemical network. The dotted horizontal lines correspond to the observed upper limit for $\mathrm{O}_{2}$ and $\mathrm{H}_{2} \mathrm{O}$ towards starless cores. The full horizontal line corresponds to the observed abundance of water towards Sgr B2

\subsubsection{The role of the $\mathrm{C} / \mathrm{O}$ ratio}

The $\mathrm{C} / \mathrm{O}$ ratio available in the gas phase is subject to large uncertainties. However, infrared observations with the ISO satellite have allowed the sampling of the abundances of oxygen and carbon found on the ice mantles towards some specific sources like W33A and the Galactic Centre (Gibbs et al. 2000). Combining these results with gas phase observations we can deduce the $\mathrm{C} / \mathrm{O}$ ratio which is found to be between 0.4 and 0.67 . The largest uncertainty lies in the actual gas phase oxygen abundance. We have performed several steady state calculations for various values of the $\mathrm{C} / \mathrm{O}$ ratio by keeping the gas phase abundance of total carbon constant and varying only the oxygen elemental abundance. Figure 7 presents the corresponding fractional abundances of $\mathrm{O}_{2}$ and $\mathrm{H}_{2} \mathrm{O}$ for a proton density $n_{\mathrm{H}}$ of $5000 \mathrm{~cm}^{-3}$. We find a region of bistability in the range $0.49-0.66$ of the $\mathrm{C} / \mathrm{O}$ ratio for the considered density. It is clearly seen that the HIP solutions lead to low values of both $\mathrm{O}_{2}$ and $\mathrm{H}_{2} \mathrm{O}$ fractional abundances.

\subsubsection{Comparison with the observations}

The HIP solutions offer a natural means to interpret, within steady state models, the low observed upper limit of molecular oxygen towards the different lines of sight. Concerning water, we find that the observed high abundance towards Sgr B2, a region of relatively low density, is equally compatible with some steady state solutions. Finally, it appears that there is no possibility to explain 


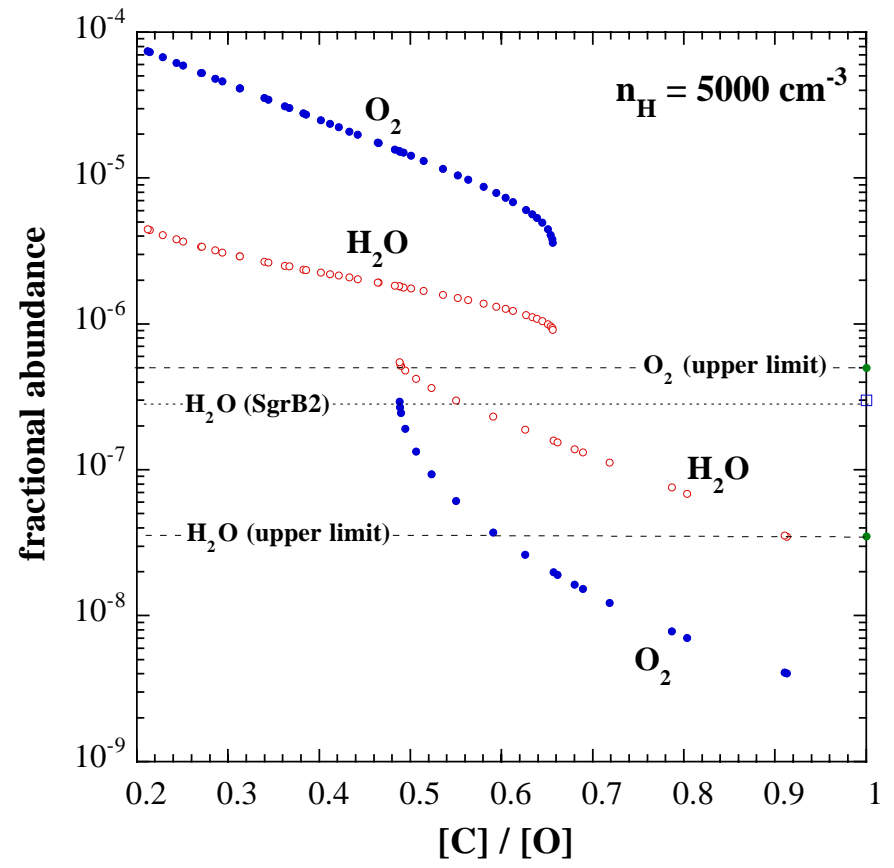

Fig. 7. Steady state stable solutions of $\mathrm{O}_{2}$ and $\mathrm{H}_{2} \mathrm{O}$ as a function of the $\mathrm{C} / \mathrm{O}$ ratio for a proton density of $5000 \mathrm{~cm}^{-3}$. The dotted horizontal lines correspond to the observed upper limit for $\mathrm{O}_{2}$ and $\mathrm{H}_{2} \mathrm{O}$. The full horizontal line corresponds to the observed abundance of water towards Sgr B2

the low abundance of water observed in high density starless cores. However, this sole interpretation is far too naive as different chemical phases are likely to be present in the large beam of the SWAS telescope. Then a combination of the different chemical phases can be considered, and would allow an explanation from the steady-state point of view of the low abundance of $\mathrm{O}_{2}$ and the large abundance of water towards Sgr B2. Another point is that HIP and LIP solutions exist, not only at steady state, but also for "early times": the two solutions usually diverge from $t>10^{6}$ yr. However, the actual temporal dependence is very dependent on the chosen initial conditions and no general conclusions may be derived. Clumpy models, such as those described by Spaans \& van Dishoeck (2001) may offer an alternative explanation although they would fail for starless cores and for sources with weak UV fields (e.g. Ophiuchus).

Concerning the lack of observations of gas phase water towards starless cores, we should keep in mind that only the ortho component of water is probed by SWAS and that no definitive conclusion about water abundance should be derived before being able to detect para- $\mathrm{H}_{2} \mathrm{O}$. Unfortunately, the lowest transition of para- $\mathrm{H}_{2} \mathrm{O}$ is at $1113 \mathrm{GHz}$, a range which is outside present capabilities of space observatories.

\section{Conclusions}

In the present study we have extensively investigated the chemistry of clump formation in low and high star formation regions and in starless cores. By comparing our theoretical predictions with observations we conclude that in dense environments $\left(n\left(\mathrm{H}_{2}\right) \geq 1000 \mathrm{~cm}^{-3}\right)$ freeze-out of gas phase species on to grains must take place in some degree. For each scenario, we find at least one model able to reproduce the majority of the observations; however, this is true only for a limited period of time, implying the clump may be transient. Its lifetime is model dependent. This result can be explained with the aid of a "cyclic model" for molecular clouds where star formation is occurring: some regions within the molecular cloud collapse into dense small clumps and form stars; once the star is born, the clumps are easily destroyed due to a total gas depletion and/or external activities such as shocks and outflows. The material they return to the ISM is then re-processed to form new clumps. Star-less cores such as TMC-1 however might not undergo a collapse phase. In this case, for the cyclic model to be valid, depletion must be the main cause of the loss of $\mathrm{H}_{2} \mathrm{O}$ and $\mathrm{O}_{2}$.

The period for which the $\mathrm{H}_{2} \mathrm{O}$ and $\mathrm{O}_{2}$ abundances determined by SWAS and the molecules observed by other telescopes, are matched reasonably well by the models, is in general - rather brief. The consequences of this brevity on the cycle-time required in molecular clouds would be severe. However, this period can be considerably extended if it is assumed that $\mathrm{CO}$ and $\mathrm{N}_{2}$ are not retained on grain surfaces during the freeze-out process, but are, in fact, returned promptly to the gas where they initiate a latephase chemistry. This chemistry persists for an appreciable fraction of the likely age of the molecular clouds; it also reflects a $\mathrm{C}: \mathrm{O}$ ratio of close to unity. A similar approach has been suggested by Bergin et al. (2000) where CO, $\mathrm{N}_{2}$ and $\mathrm{CH}_{4}$ are allowed to evaporate because the temperature of the dust grains is maintained at $30 \mathrm{~K}$. They, too, find that this approach reproduces the low abundances of $\mathrm{H}_{2} \mathrm{O}$ and $\mathrm{O}_{2}$. The main difference in our approach is that, by retaining $\mathrm{CH}_{4}$ on the grains, we do not increase the abundances of hydrocarbons and cyanopolyynes in the gas phase (Nejad \& Millar 1988). While no attempt can be made to produce a precise fit for any particular object, given the nature of the SWAS observations, it is clear that the overall results of the SWAS mission can be interpreted in terms of time-dependent chemistry, and that this interpretation is strengthened if selective freeze-out occurs.

We have also shown that the range of physical conditions considered is appropriate to the occurrence of bistable steady state solutions. The so-called HIP phase offers a natural explanation for low abundances of $\mathrm{O}_{2}$ and $\mathrm{H}_{2} \mathrm{O}$, even at steady state. Also, the intrinsic uncertainties in the chemical network, even for the simple molecular species discussed here, are compatible with a bistable behaviour. The possibility that the two chemical phases contribute to the observed column density is most likely and a combination of different chemical phases still may explain both the large abundance of water towards Sgr B2 and the ubiquitous low abundance of molecular oxygen. The upper limit found for gas phase $\mathrm{H}_{2} \mathrm{O}$ in starless cores is much more striking. However, the SWAS results refer only to the ortho form and a definitive conclusion 
should wait for a comparable study of the para form of water. This is still for the future.

Acknowledgements. We thank the referee for constructive comments that helped to improve on an earlier version of this paper. SV and DAW thanks PPARC for financial support. ER, DAW and GPdF acknowledge the TMR "Astrophysical Chemistry" \#ERBFMRXCT970132.

\section{References}

Bergin, et al. 2000, ApJ, 539, L129

Bergin, E. A., Ungerechts, H., Goldsmith, P. F., et al. 1997, ApJ, 482, 267

Charnley, S., Dyson, J. E., Hartquist, T. W., \& Williams, D. A. 1988, MNRAS, 235, 1257

Davidsson, J., \& Stenholm, L. G. 1990, A\&A, 230, 504

Gerin, M., Falgarone, E., Joulain, K., et al. 1997, A\&A, 318, 579

Gibbs, E. L., et al. 2000, ApJ, 536, 347

Goldsmith, et al. 2000, ApJ, 539, L123

Greaves, J. S., White, G. J., Ohishi, M., Hasegawa, T., \& Sunada, K. 1992, A\&A, 260, 381

Gredel, R. 1990, in Molecular Astrophysics: A Volume Honouring A. Dalgarno, ed. T. W. Hartquist (Cambridge University press), 305

Gwenlan, C., Ruffle, D. P., Viti, S., Hartquist, T. W., \& Williams, D. A. 2000, A\&A, 354, 1127

Jensen, M. J., Bilodeau, R. C., Safvan, C. P., Seiersen, K., \& Andersen, L. H. 2000, ApJ, 543, 764

Habing, H. J. 1968, Bull. Astron. Inst. Netherlands, 19, 421

Hartquist, T. H., \& Williams, D. A. 1990, MNRAS, 247, 343

Harwit, M., Neufeld, D. A., Melnick, G. J., \& Kaufman, M. J. 1998, ApJ, 497, L195

Hirahara, Y., Suzuki, H., Amamoto, S., et al. 1992, ApJ, 394, 539

Howard, C. J., Smith, \& I. W. H. 1981, J. C. S. Faraday Trans. $2(77), 997$

Hüttemeister, S., Wilson, L., Henkel, C., \& Mauersberger, R. 1993, A\&A, 276, 445

Le Bourlot, J., Pineau des Forêts, G., Roueff, E., \& Schilke, P. 1993, ApJ, 416, L87

Le Bourlot, J., Pineau des Forêts, G., \& Roueff, E. 1995, A\&A, 297, 251

Lee, H.-H., Roueff, E., Pineau Des Forêts, G., et al. 1998, A\&A, 334,1047
Linke, R. A., Stark, A. A., \& Frerking, M. A. 1981, ApJ, 243, 147

Maréchal, P., Viala, Y. P., \& Benayoun, J. J. 1997, A\&A, 324, 221

Mc Call, B. J. , Geballe, T. R. , Hinkle, K. H., \& Oka, T. 1999, ApJ, 522, 338

Meyer, D. M., Jura, M., \& Cardelli, J. A. 1998, ApJ, 493, 222

Millar, T. J., \& Nejad, L. A. M. 1985, MNRAS, 217, 507

Millar, T. J., Farquhar, P. R. A., \& Willacy, K. 1997, A\&AS, 121,139

Moneti, A., Cernicharo, J., \& Pardo, J. R. 2001, ApJL, in press

Nejad, L. A. M., \& Williams, D. A. 1992, MNRAS, 255, 441

Nejad, L. A. M., \& Millar, T. J. 1988, MNRAS, 230, 79

Neufeld, et al. 2000, ApJ, 539, L111

Nisini, B., Benedettini, M., Giannini, T., et al. 2000, A\&A, 360,297

Norman, C., \& Silk, J. 1980, ApJ, 238, 158

O'neill, P. T., \& Williams, D. A. 1999, Ap\&SS, 266, 539

Rawlings, J. M. C., Hartquist, T. W., Menten, K. M., \& Williams, D. A. 1992, MNRAS, 255, 471

Ruffle, D. P., Hartquist, T. W., Taylor, S. D., \& Williams, D. A. 1997, MNRAS, 291, 235

Ruffle, D. P., Hartquist, T. W., Caselli, P., \& Williams, D. A. 1999, MNRAS, 306, 691

Shah, M. B., \& Gilbody, H. B. 1982, J. Phys. B, 15, 3441

Smith, I. W. H., \& Stewart, D. W. A. 1994, J. Chem. Soc., Faraday Trans., 90, 3221

Snow, T. P., \& Witt, A. N. 1996, ApJ, 468, 65L

Shutte, W. A. 1999, in Laboratory Astrophysics and Space Research, ed. P. Ehrenfreund, \& H. Kochan, in press

Spaans, M., \& van Dishoeck, E. F. 2001, ApJ, in press

Takano, S., Masuda, A., Hirahara, Y., et al. 1998, A\&A, 329, 1156

Vandenbussche, B., et al. 1999, A\&A, 346, L57

Vejby-Christensen, L., Andersen, L. H., Heber, O., et al. 1997, ApJ, 483, 531

Viti, S., Williams, D. A., \& O'neill, P. T. 2000, A\&A, 354, 1062

Viti, S., \& Williams, D. A. 1999, MNRAS, 305, 755

Willacy, K., Williams, D. A., \& Duley, W. W. 1994, MNRAS, 267, 949

Willacy, K., \& Williams, D. A. 1993, MNRAS, 260, 635

Williams, D. A., \& Hartquist, T. W. 1984, MNRAS, 210, 141

Williams, T. L., Adams, N. G., Babcock, L. M., Herd, C. R., \& Geoghegan, M. 1996, MNRAS, 282, 413 